\title{
EDWARD WYLLYS ANDREWS IV \\ (1916-1971)
}

por ALBERTO RUZ LHUILLIBR

En mi último viaje a Yucatán, a fines de diciembre de 1970, llamé a casa del doctor Andrews con el propósito de ir a saludarlo, como acostumbraba hacer cada vez que regresaba por unos días a Mérida. No lo encontré: acababa de ser transportado a los Estados Unidos, muy enfermo, para que le hicieran una serie de exámenes médicos. Supe más tarde que seguía grave, que había sido operado y finalmente me enteré por la prensa de su fallecimiento. Poco después de enviar mi pésame a sus familiares, una carta de Robert Wauchope, Director del Middle American Research Institute, de la Universidad de Tulane, de cuya institución dirigía el doctor Andrews el programa de investigaciones en Yucatán, me hacía saber que al sentirse muy grave y prever el desenlace de su enfermedad, Bill había pedido que su amigo Alberto Ruz escribiera su nota necrológica para Estudios de Cultura Maya.

Cuando la muerte toca a alguna persona, familiar o amigo, de menor edad que uno, la impresión es más aguda que cuando se trata de alguien mayor. Se siente como que algo indebido ha sucedido, que el destino no respetó la lógica prioridad cronológica, y la fragilidad de nuestra propia existencia se hace más patente. Así lo percibí con la muerte de Bill quien solía llamarse a sí mismo "the old Bill", expresión que cada vez que la repetía delante de mí me recordaba que yo le llevaba diez años.

Coincidimos en Mérida durante varios años; procuré en mi carácter de Jefe de la Oficina de Monumentos Prehispánicos en Yucatán (INAH) facilitarle los trámites burocráticos oficiales que implicaba su labor en las exploraciones de Dzibilchaltún; ayudarle a conseguir el personal técnico para las obras de restauración; contestar alguna que otra pregunta que me hiciera en cuanto a detalles relativos a la reconstrucción o conservación de los monumentos. Pero nuestras re- 
laciones fueron más amistosas que oficiales, y tuvieron como marcos habituales el portal de su residencia de Mérida, o en los fines de semana la terraza de la casa que alquilaba en la playa de Chicxulub, contigua a la mía.

Difícilmente, aun en la intimidad, se alejaba del tema arqueológico, su obsesión y al parecer la mayor motivación de su vida. Creo sinceramente que su gran amor fue la arqueología maya, el estudio de sus problemas, la búsqueda en la selva de Campeche y Quintana Roo, en los montes de Yucatán, en los litorales de la península, de antiguas ciudades desconocidas, de monumentos que pese a su estado de destrucción conservaran algunos elementos susceptibles de orientarlo para establecer relaciones estilísticas, secuencias cronológicas, influencias interculturales. Tenía permanentemente en la mente alguna cuestión arqueológica que le preocupaba, y se percibía en su compañía una ansia de hallar solución. Era tan intensa y constante su preocupación por resolver los problemas de la historia antigua de los mayas, que a su lado siempre me sentía un poco como un arqueó$\log$ a aficionado.

Quizá se debiera una vocación tan absoluta y absorbente al hecho de que se inició a muy temprana edad. Sólo tenía 17 años cuando, según se cuenta, se presentó a Chichen-Itzá ante el doctor Morley, entonces director del Proyecto de Exploraciones en Yucatán de la Carnegie Institution de Washington, diciéndole que ya sabía sobre los jeroglíficos mayas todo lo que el propio Morley había publicado y que deseaba aprender más. No hay por qué dudar de la veracidad de la anécdota, ya que pocos meses después (1934) se publicaba, en American Anthropologist, su primer trabajo sobre el tema bastante arduo del "Glifo $\mathrm{X}$ en las Series Suplementarias de las Inscripciones Mayas". Este trabajo había sido escrito dos años antes, es decir cuando Andrews tenía sólo 16 años.

La significación de los glifos que integran lo que llamamos Serie Suplementaria, relativa al ciclo de las 9 deidades acompañantes y del cómputo lunar, lo preocupó particularmente, y trató el tema en varios trabajos $(1934,1936,1938,1951)$. Sobre esta cuestión versó su tesis de Maestría (1938), pero no sólo se interesó por signos específicos, sino también por asuntos más amplios de la epigrafía maya, y escribió síntesis sobre cronología y astronomía en el área maya (1940a). También discutió y en alguna forma criticó ciertos aspectos de los trabajos de Thompson (1958) y Zimmermann (1959f).

También muy joven inició largos reconocimientos en el área maya, recorriendo numerosos sitios, levantando planos, tomando fotografías 
y apuntando todos los datos, arquitectónicos y otros, susceptibles de dar algo de luz sobre los sitios que visitaba. Desde 1934, y principalmente en los años de 1939 a 1941, recorrió Quintana Roo, Campeche y parte de Yucatán. Gran parte de los resultados de sus reconocimientos fueron publicados por la Institución Carnegie en "The archaeology of Southwestern Campeche" (1943), y en varios "Year Book" de la misma institución (números 39 y 41).

Durante la Segunda Guerra Mundial, prestó servicios en la marina norteamericana, y fue condecorado por su actuación, ligada a la Oficina de Servicios Estratégicos, en África, Italia, Francia y Alemania. Más tarde siguió prestando servicios durante varios años en la misma dependencia y en la Agencia Central de Inteligencia.

Retornó al campo de la cultura maya, primero con una reseña sobre una versión nueva del Popol Vub (1950), luego con su tema preferido de las Series Suplementarias (1951) que desarrolló aprovechando una beca Guggenheim. Entre los años de 1952-1956 realizó dos expediciones en sitios de la costa oriental de la península de Yucatán, en que visitó y estudió numerosos sitios, entre ellos Xcaret, Paalmul, Chakalal y Punta Chile.

En 1956 inició las exploraciones en un sitio cercano a Mérida, el que iba a convertirse en el centro de sus trabajos durante 12 años y sin duda el sitio al que dedicó mayores esfuerzos e interés profesional: Dzibilchaltún. Sus investigaciones fueron auspiciadas por el Middle American Research Institute, de la Universidad de Tulane, y la National Geographic Society de Washington; con donativos adicionales de la National Science Foundation y la American Philosophical Society. No es éste el momento de analizar los resultados de estas exploraciones, los que además no han sido aún publicados, pero de las informaciones parciales (1959, 1960, 1961, 1962, 1965, 1968) se desprende la gran importancia de Dzibilchaltún, como centro ceremonial y al parecer urbano de enorme extensión, fuerte densidad de construcciones y larguísima ocupación.

Durante los años del proyecto de Dzibilchaltún participó en la exploración de la gruta de Balankanché, santuario tolteca al dios de la lluvia, en la cercanía de Chichén-Itzá; de la cueva de Chaac, a poca distancia de Kabah; y de depósitos del periodo Pre-clásico en la isla de Cancún, en la costa caribeña. El resultado de estas exploraciones fueron oportunamente publicadas $(1965,1970,1971)$.

Los últimos años de su vida, los dedicó a una región arqueológicamente no explorada, aunque suficientemente conocida para que sepamos que se trata de un área de convergencia a la que llegaron 
marcadas influencias del Petén, al sur, y de los Chenes, al norte. Esta región denominada Río Bec, por llevar este nombre un sitio característico del estilo de la misma, se sitúa al sureste del Estado de Campeche y suroeste del territorio de Quintana Roo. El sitio de Becan fue el que Andrews exploró más intensamente, y en el que llevó a cabo obras de restauración. Los resultados de sus trabajos quedaron preparados para su publicación.

Problemas de carácter general le interesaban tanto como los muy específicos de un sitio o de un periodo determinado, y los trató en diferentes publicaciones. Entre ellos se destacan, por su importancia entre las investigaciones actuales, el de la emergencia de la civilización maya en el medio selvático, aparentemente poco propicio de las tierras bajas $(1970,1971)$, y el de su colapso en el área central, no suficientemente aclarado aún (1971).

Buscar elementos a estudiar, clasificarlos, analizarlos, compararlos, deducir conclusiones, constituían para Bill Andrews una necesidad irrefrenable. Cuando acababa de descubrir al Yucatán arqueológico, percatándose de las numerosas especies de serpientes que allí existían, no resistió a la tentación de colectar ofidios, estudiarlos y publicar sus observaciones $(1936,1937)$. Más tarde, cuando sentía la urgencia de descansar del agobiador trabajo de campo de Dzibilchaltún, recorría con sus hijos las playas de la costa oriental de la península yucateca, pero su descanso consistía en recoger conchas de moluscos, centenares, millares de ellas. Publicó un trabajo sobre el uso y la distribución arqueológica de moluscos en las tierras bajas mayas (1969), y dejó un impresionante material perfectamente clasificado, inventario virtualmente exhaustivo de las conchas actuales yucatecas. Su próximo estudio, en las ciencias naturales, iba a ser el de las orquídeas, de las que había iniciado ya el catálogo.

El doctor Andrews fue un incansable trabajador científico. Creo que íntimamente sabía que su vida no sería muy larga, y que era imprescindible para él aprovechar al máximo los días de su existencia para conocer más de la historia y de la cultura de los mayas, así como de la fauna y de la flora del país que había escogido a los 17 años, como marco futuro de sus preocupaciones científicas, de su insaciable sed de conocimientos.

Su prematura desaparición dejó un gran vacío en el campo de la investigación mayista y más específicamente de la arqueología de la península yucateca. Sus amigos extrañaremos su trato cordial, su generosa hospitalidad, su sonrisa burlona, la penetrante agudeza de su mirada, su personalidad a la vez discreta y brillante. 


\section{BIBLIOGRAFIA}

Reunida por Robert Wauchope y Joann M. Andrews

1934 "Glyph X of the Supplementary Series of the Maya Inscriptions." American Antbropologist, vol. 36, Washington, D. C., pp. 345-354.

1936 a "Notes on Glyph G of the Maya Inscriptions." Maya Research, vol. 3, pp. 306-308.

1936b (con Karl P. Schmidt) "Notes on Snakes from Yucatan." Field Museum of Natural History, Zoological Series, núm. 20, pp. 167-187.

1937 a "Notes on Snakes from the Yucatan Peninsula." Field Museum of Natural History, Zoological Series, núm. 20, pp. 355-359.

$1937 \mathrm{~b}$ A survey of the maya stelae of the state of Yucatan (manuscrito).

1938a "Glyphs Z and Y of the Maya Supplementary Series." American Antiquity, vol. 4, Washington, D. C., pp. 30-35.

1938 b "The Phonetic Value of Glyph C of the Maya Supplementary Series." American Anthropologist, vol. 40, Washington, D. C., pp. 755-758.

1938c The maya supplementary series. Distinction Thesis. Harvard College (manuscrito).

1938d "Some New Material from Coba, Quintana Roo, Mexico." Ethnos, núm. 3, pp. 33-46.

1939 "A Group of Related Sculptures from Yucatan." Carnegie Institution of Washington, Contributions to American Antbropology and History, núm. 26.

1940a "Chronology and Astronomy in the Maya Area", in The Maya and their Neighbors, pp. 150-161. D. Appleton-Century Company, New York

1940b "Exploration in Southwestern Campeche, Tabasco, and the Department of Peten". Carnegie Institution of Wasbington, Year Book 39, pp. 267-269.

1941a "An Ethnological Note from Cilvituk, Southern Campeche." Carnegie Institution of Wasbington, Notes on Middle American Archaelogy and Etbnology 5.

1941b "The Ruins of Culuba, Northeastern Yucatan." Carnegie Institution of Wasbington, Notes on Middle American Archaeology and Etbno$\log y$, núm. 3.

1941c "Pustunich, Campeche; Some Further Related Sculptures", en Los Mayas Antiguos, pp. 125-235. El Colegio de México, México.

1942 a "The Inscription on Stela 38, Piedras Negras, El Peten, Guatemala." American Antiquity, vol. 7, pp. 364-368.

1942b "Yucatan: Architecture." Carnegie Institution of Washington, Year Book, núm. 41, pp. 257-263.

1943 "The Archaeology of Southwestern Campeche." Carnegie Institution of Wasbington, Contributions to American Antbropology and History, vol. VII, núm. 40, pp. 1-100.

1950 Reseña: "Popol Vub: The Sacred Book of the Ancient Qiché", versión inglesa Delia Goetz y Slyvanus G. Morley (editores), en Scientific Montbly, núm. 71, p. 281. 
1951 "The Maya Supplementary Series", en The Civilizations of Ancient America, Proceedings of the 29th International Congress of Americanists, núm. 1, editado por Sol Tax, pp. 123-141, University of Chicago Press, Chicago.

1956 "We Sought a lost City." The Times Picayune, Dixie Roto Magazine, junio 24, núm. 2, pp. 8-9.

1958 a "Discussion of Research in Maya Hieroglyphic Writing" by J. E. S Thompson, Pan American Union, Social Science Monograph, núm. 5, pp. 58-60.

1958b "Light on the Ancient Maya." Explorer's Journal, núm. 36, pp. 8-10.

1958c "A Revision of Some Dates on the Hieroglyphic Stairway, Copan, Honduras." Middle American Research Institute, Tulane University, núm. 26 , pp. $1-8$.

1959a "Dzibilchaltún, la Ciudad Perdida de los Mayas." Excélsior, enero, pp. 14-17.

1959b "Dzibilchaltun, Lost City of the Maya." National Geograpbic Magazine, núm. 115, pp. 90-109.

1959c "A Lost Maya City Arises." Explorer's Journal, núm. 37, pp. 11-17.

1959d "Preliminary Progress Report on 1958-1959 Field Season Dzibilchaltun Program." Middle American Research Institute. University Tulane. New Orleans.

1959e "Progress Report on Two Seasons' Work at Dzibilchaltun, Northwestern Yucatan, Mexico: 1956-57, 1957-58." National Geograpbic Society (edición en offset).

$1959 f$ Reseña de: "Die Hieroglyphen der Maya-Handschriften", Günter Zimmermann, en American Antiquity, núm. 24, pp. 441-443.

1960a "Archaeological Excavations in Yucatan." American Pbilosopbical Society, Year Book 1959, pp. 466-467.

1960b "Excavations at Dzibilchaltun, Northwestern Yucatan, Mexico." Proceedings of the American Pbilosopbical Society, núm. 104, pp. 254-265.

1960c Reseña de: "Middle American Anthropology: Special Symposium of the American Anthropological Association", G. R. Willey, E. Z. Vogt y Angel Palerm, en American Journal of Arcbaeology, núm. 64, pp. 216-217.

1961a "Excavations at the Gruta de Balankanche, 1959." Middle American Research Institute, Miscelaneous Series, núm. 11, pp. 28-40.

1961b "Preliminary Report on the 1959-60 Field Season, National Geographic Society-Tulane University Dzibilchaltun Program." Middle American Research Institute, Miscellaneous Series, núm. 11, pp. 1-27.

1961c Reseña de: "Prehistoric Ceramics and Settlement Patterns in Quintana Roo, Mexico", W. T. Sanders, en American Antiquity, núm. 27, pp. 123-124.

1962a "Excavaciones en Dzibilchaltún, Yucatán, 1956-1962", en Estudios de Cultura Maya, vol. II, pp. 149-183.

1962b "Excavations at Dzibilchaltun, Yucatan, 1956-1962." Traducción del español $-1962 a$ -

1963 (con Anna O. Shepard) "Imitation Jade Ornaments From Dzibil- 
chaltun, Yucatan." Carnegie Institution of Washington, Notes from a Ceramic Laboratory 3.

1964 Reseña de "A Catalog of Maya Hieroglyphs", J. Eric S. Thompson, en Archaeology, núm. 17, p. 288.

1965a "Archaeology and Prehistory in the Northern Maya Lowlands: Introduction', en Handbook of Middle American Indians, vol. 2, editado por Gordon R. Willey, pp. 288-330. University of Texas Press, Austin.

1965b "Explorations in the Gruta de Chac, Yucatan, Mexico." Middle American Research Institute, Tulane University, Publicación núm. 31, pp. 1-21.

1965 c "Progress Report on the 1960-1964 Field Seasons, National Geographic Society-Tulane University Dzibilchaltun Program." Middle American Research Institute, Tulane University, Publicación núm. 31, pp. 23-67.

1966 Reseña de: "Prehistoric Maya Settlements in the Belize Valley." G. R. Willey, W. R. Bullard, Jr., J. B. Glass, J. C. Gifford y otros, en American Antbropologist, núm. 68, 1959-1960.

$1967 a$ (con Stanley H. Boggs) "An African Art Object in Apparently Early Archaeological Context in El Salvador: a Caveat to the Diffusionist", en Etnos, núm. 32, pp. 18-25.

1967b Reseña de: "Early Cultures and Human Ecology in South Coastal Guatemala", Michael D. Coe y Kent V. Flannery, en Science núm. 158, pp. 364-365.

1968a "Dzibilchaltun, a Northern Maya Metropolis", en Archaeology, vol. 21, núm. 1, pp. 36-47.

$1968 \mathrm{~b}$ "The Archaeology of the Northern Maya Area (Introduction)", en Archaeology, vol. 21, núm. 1, p. 47.

1968 c "National Geographic Society-Tulane University Program of Archaeological Research at Dzibilchaltun, Yucatan, Mexico." National Geographic Society, Research Reports, 1963 Projects, pp. 241-242.

1968d (con George E. Stuart) "The Ruins of Ikil, Yucatan, Mexico." Middle American Research Institute, Tulane University, Publicación núm. 31, pp. 69-80.

1968 e "Torre Cilíndrica de las Ruinas de Puerto Rico", Campeche. INAH, Boletin 31, pp. 7-13.

1969a "The Archaeological Use and Distribution of Mollusca in the Maya Lowlands." Middle American Research Instituie, Tulane University, Publicación núm. 34.

1969b Reseña de: "Archaeological Explorations in El Peten, Guatemala". Ian Graham, en American Antiquity 34, p. 194.

1970a "Balankanche, Throne of the Tiger Priest." Middle American Research Institute, Tulane University, Publicación núm. 32.

1970 b "The Emergence of Civilization in the Maya Lowlands." Trabajo preparado para presentarse en el symposium núm. 47 en Burg Wartenstein de la Wenner-Gren Foundation for Anthropological Research. Mimeografiado.

1970c "The Development of Maya Civilization After Abandonment of the Southern Cities." Trabajo presentado en el Seminar on the Collapse 
of Maya Southern Lowland Civilization, en School of American Research, Santa Fe, New Mexico.

1971a "The Emergence of Civilization in the Maya Lowlands", en Observations on the Emergence of Civilization in Mesoamerica. Editado por Robert F. Heizer y John A. Graham. Contributions of the University of California Archaeological Facility num. 11, pp. 85-96.

1971b "Pre-Agricultural or Early Ceramic Remains on Yucatan Coasts." American Pbilosopbical Society, Year Book 1970, pp. 540-541.

1971c Reseña de: "La Costa de Campeche en los Tiempos Prehispánicos: Prospección Cerámica y Bosquejo Histórico", Alberto Ruz L., en American Antbropologist, núm. 73, pp. 424-425.

s. f. (con Irwin Rovner) "Archaeological Evidence on Social Stratification and Commerce in the Northern Maya Lowlands: Two Masons' Tool Kits from Muna and Dzibilchaltun, Yucatan." Middle American Research Institute, Tulane University.

s. f. A Preliminary Study of the Ruins of Xcaret, Quintana Roo, México. En preparación. 


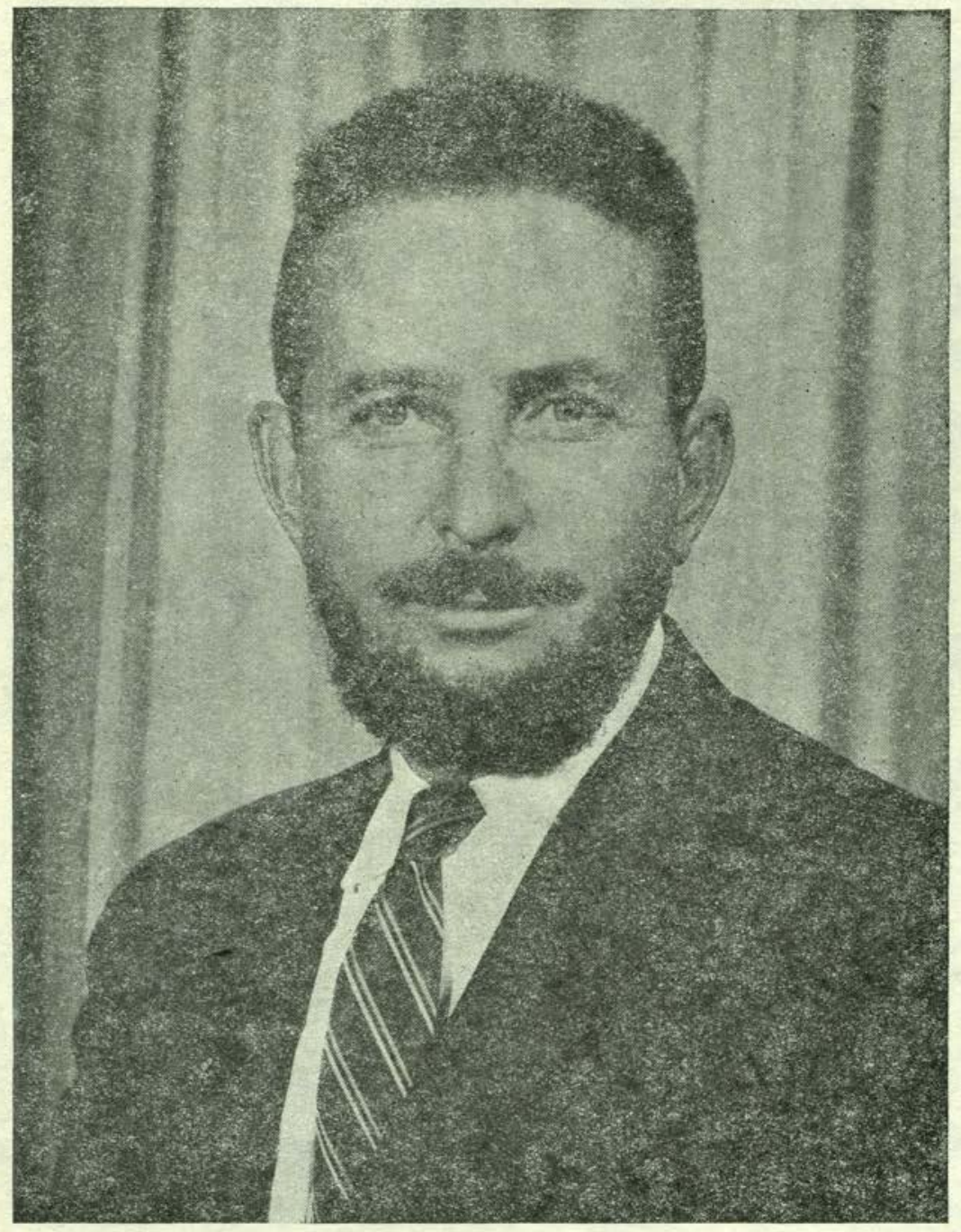

Edward Wyllys Andrews IV

Estudios de Cultura Maya. Vol. IX, 1973

Instituto de Investigaciones Filológicas/

Facultad de Filosofía y Letras, UNAM 